\title{
PLASMA FLUCTUATIONS IN CROSSED ELECTRIC AND MAGNETIC FIELDS*
}

BY

\author{
H. W. BATTEN, ${ }^{1}$ H. L. SMITH ${ }^{1}$ AND H. C. EARLY ${ }^{1}$
}

\section{ABSTRACT}

An experimental study has been made of the high-amplitude electrical fluctuations associated with a gas discharge in a strong magnetic field. Hydrogen, helium, argon, and nitrogen gas were used at pressures from 0.3 to 100 microns. The power spectrum on plasma probes was investigated for various values of magnetic field, gas pressure, and power input to the discharge. For the range of frequencies studied ( 0.5 to 4000 megacycles per second), the spectrum shows a continuous high level that increases in amplitude with decreasing pressures at all frequencies and increases with increasing magnetic field at low frequencies. A sharp dip in the spectrum was observed near the ion cyclotron frequency for discharges in hydrogen and helium. The velocity and direction of propagation for the low frequency fluctuations were determined by cross-correlating the potential variations on two neighboring probes. These fluctuations appear to propagate in the direction of the electron drift and at a velocity somewhat less than the Lorentz drift velocity.

\section{INTRODUCTION}

A low-pressure, electrical discharge transverse to a strong magnetic field is characterized by large-amplitude, plasma fluctuations (1). ${ }^{2}$ These spontaneous electrical fluctuations are hundreds of volts in amplitude, and play an important role in the scattering, diffusion, and ionizing processes of the gas (2). Without the magnetic field, the spectrum of the fluctuations consists of discrete frequencies (3), while the presence of the magnetic field suppresses these isolated frequencies and produces broad frequency ranges of high-intensity noise.

The present paper outlines an experimental study of the spontaneous fluctuations associated with a glow discharge in a strong transverse magnetic field. The objective is to answer the following questions:

1. How is the power in the fluctuations distributed in frequency? How does this distribution depend on the pressure, magnetic field, and type of gas?

2. Do these fluctuations propagate in the medium? If so, in what direction do they propagate, and how fast?

3. Do these fluctuations amplify or attenuate as they propagate?

* This research was sponsored by the Office of Ordnance Research, U. S. Army.

1 Engineering Research Institute of the University of Michigan, Ann Arbor, Mich.

2 The boldface numbers in parentheses refer to the references appended to this paper. 
All the experiments used the cylindrical electrode geometry shown in Fig. 1. The anode was 8 in. in diameter; part of this anode was made of copper screen so the discharge could be seen during operation. The cathode consists of the two disks in the center of the cylinder, the cathode action being similar to that in a Phillips Ionization Gage.

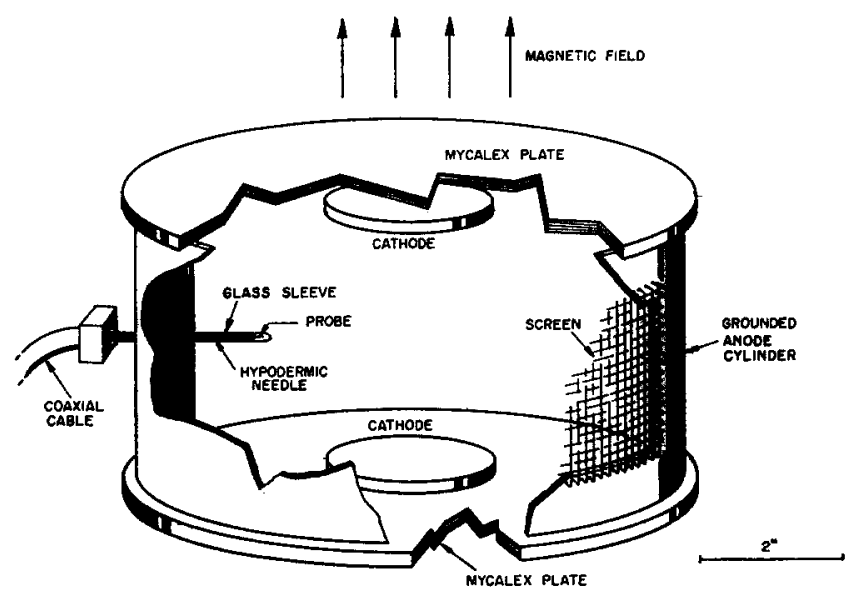

FIG. 1. Electrode structure used in the experiments, showing probe in place.

The entire assembly of Fig. 1 was located inside a large vacuum chamber. A glass window on one side of the chamber provided good visibility of the discharge. To minimize contamination of the gas, a fast vacuum pump (100 liters per second) was used, and gas was bled into the chamber at a rate sufficient to maintain the desired equilibrium pressure. The undesirable effects due to heating and outgassing of the dielectric plates were avoided by operating the discharge for only short intervals.

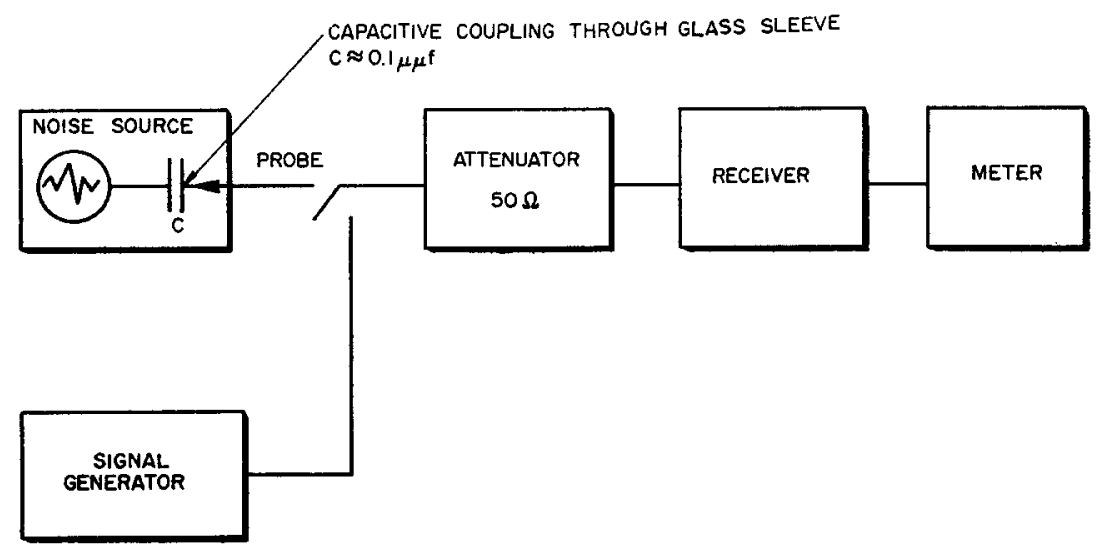

FIG. 2. Block diagram of instrumentation circuit. 
All the data were taken near the close of a three to five second operating interval.

Figure 2 is a block diagram illustrating the method used for obtaining the spectral data. The plasma probe consisted of a short tip of fine wire projecting from the end of a hypodermic needle as illustrated in Fig. 1. The fine wire passing through the hollow needle formed a 50-ohm coaxial line. This probe assembly was connected to a terminated length of 50-ohm R.G. cable so the load impedance seen by the probe was the same at all frequencies. The projecting wire tip which formed the active part of the probe was covered with an insulating glass sleeve, and all the voltage induced in the probe was due to capacitive coupling. ${ }^{3}$ The $50-\mathrm{ohm}$ cable from the probe was connected to a superheterodyne receiver through an isolation attenuator. The output of this receiver was metered so that the noise input in the passband of the receiver could readily be determined by using a signal generator as shown in Fig. 2.

\section{CHARACTERISTICS OF A "REVOLVING PLASMA" DISCHARGE $(4,5)$}

The presence of the axial magnetic field gives the electrode geometry used in these experiments a resemblance to a smooth-bore magnetron. The ions and electrons have a large circumferential component of drift velocity and the resulting momentum exchange with neutral gas molecules causes all of the gas in the region to revolve at high velocity. This high velocity "wind" can be observed by mounting a small flag of mica or glass cloth which will stand out horizontally in the air stream. Visually, the discharge in nitrogen is pale blue and quite transparent, the region between the cathode discs being more densely luminous than the rest of the plasma.

The discharge was usually operated at a current of $0.5 \mathrm{amp}$. With a gas pressure of $0.01 \mathrm{~mm}$. of $\mathrm{Hg}$, and a magnetic field of 3500 gauss, the average voltage across the electrodes was approximately 1500 volts. The power supply voltage was 8000 volts, most of the voltage drop occurring in a non-inductive resistance in series with the cathode lead. Thus, the discharge operated at essentially constant current conditions.

\section{THE POWER SPECTRUM OF THE DISCHARGE}

A great many parameters affect the experimental study of the power spectrum. They include the type of gas, gas pressure, magnetic field strength, discharge current, type of probe, position and orientation of the probe, and many others. A survey of the influence of these factors has been made, and certain features are especially interesting.

Figure 3 shows the power spectrum of the voltage fluctuation on a probe in a hydrogen discharge. The striking characteristic of this curve is the sharp dip at the ion cyclotron frequency and the rise in the curve above and below that frequency. It is important to note that

${ }^{3}$ The merits and shortcomings of various types of probes are discussed in the Appendix. 


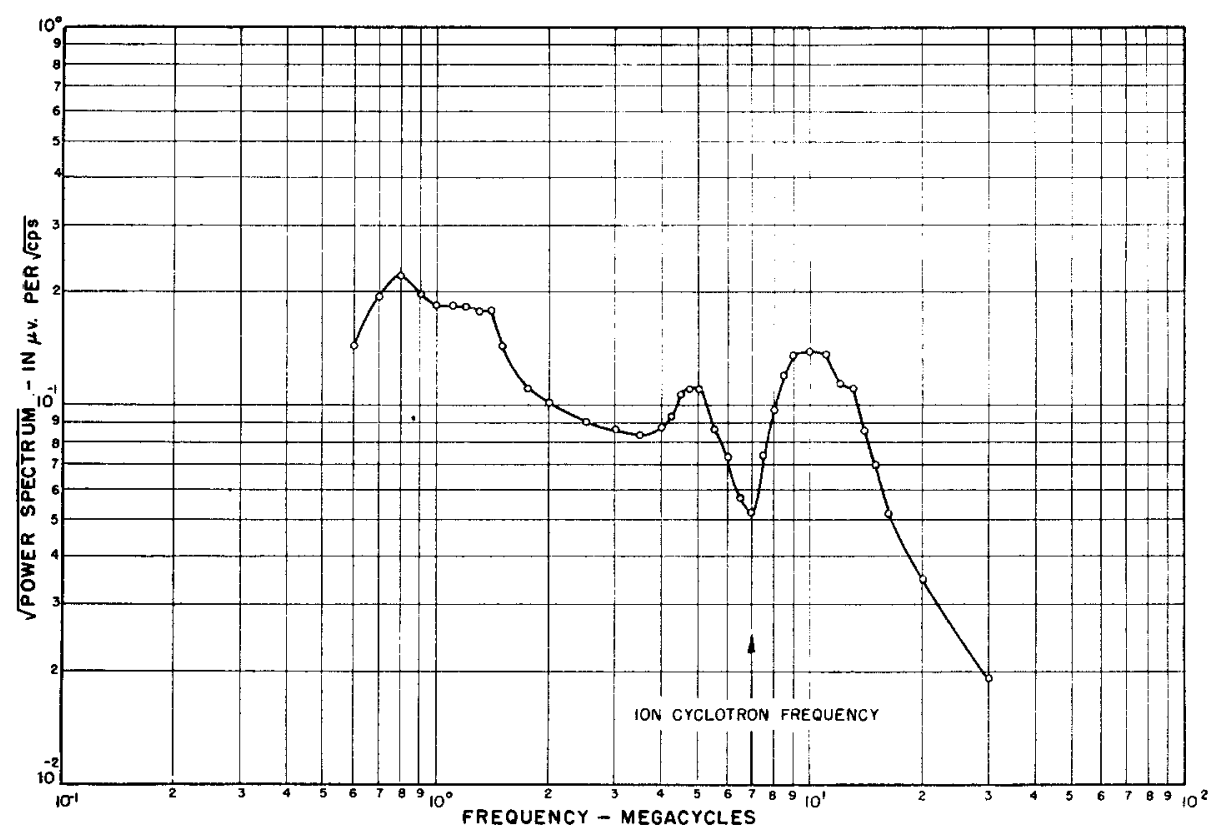

FIG. 3. Hydrogen discharge in a magnetic field.

$B=4550$ gauss
$I=0.5$ amp.

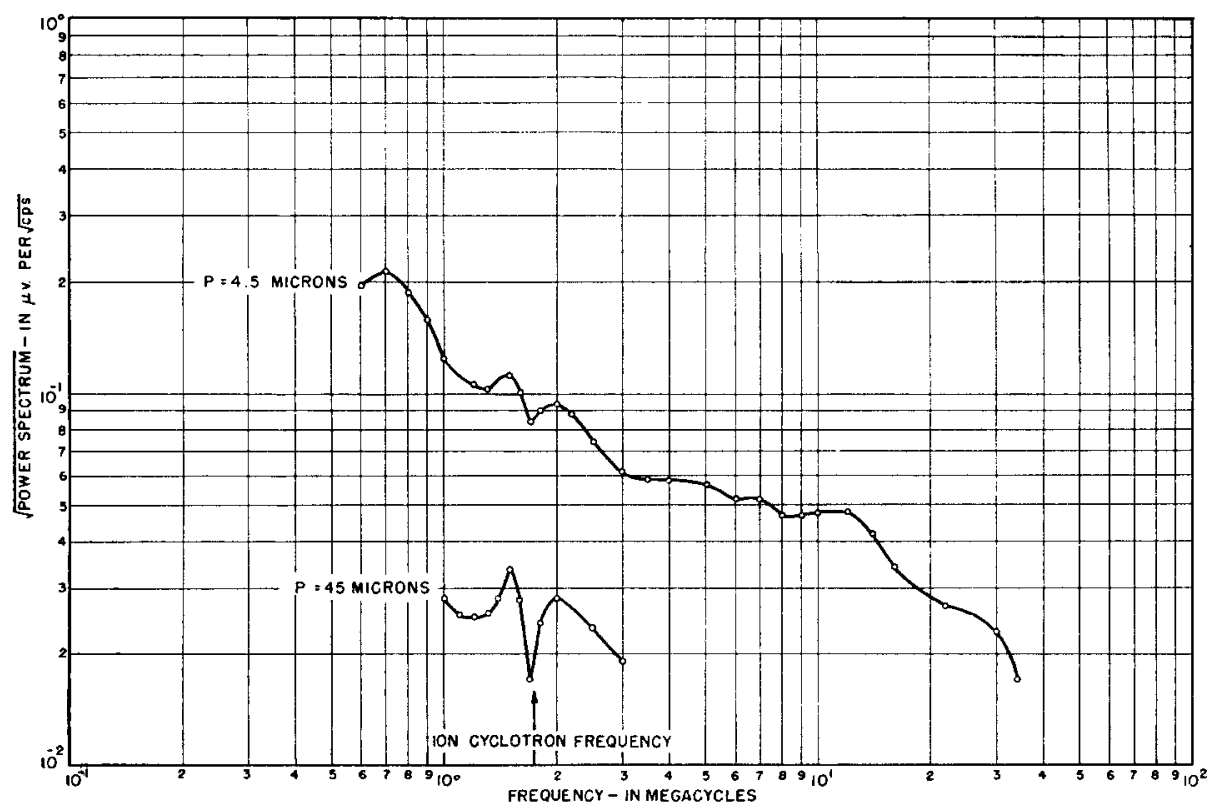

FIG. 4. Helium discharge in a magnetic field.

$$
\begin{aligned}
B & =4550 \text { galls } \\
I & =0.5 \text { aml). }
\end{aligned}
$$


the cyclotron frequency is computed assuming an ionized atom; if the ionized molecules are present in the gas, their effect is certainly much less pronounced in the spectrum. Two other features in Fig. 3 are of interest. (1) Near 800 kilocycles per second there appears to be a broad resonance. It is likely that this resonance is to be identified with ions oscillating in a potential minimum as discussed by Ballantine, and later Cobine (6). (2) The power spectrum seems to decrease markedly near 15 megacycles per second.

The cyclotron dip, similar to that of Fig. 3, is shown in Fig. 4 where the power spectrum for a helium discharge is given. It is of interest to note that the dip is less pronounced for helium than for hydrogen.

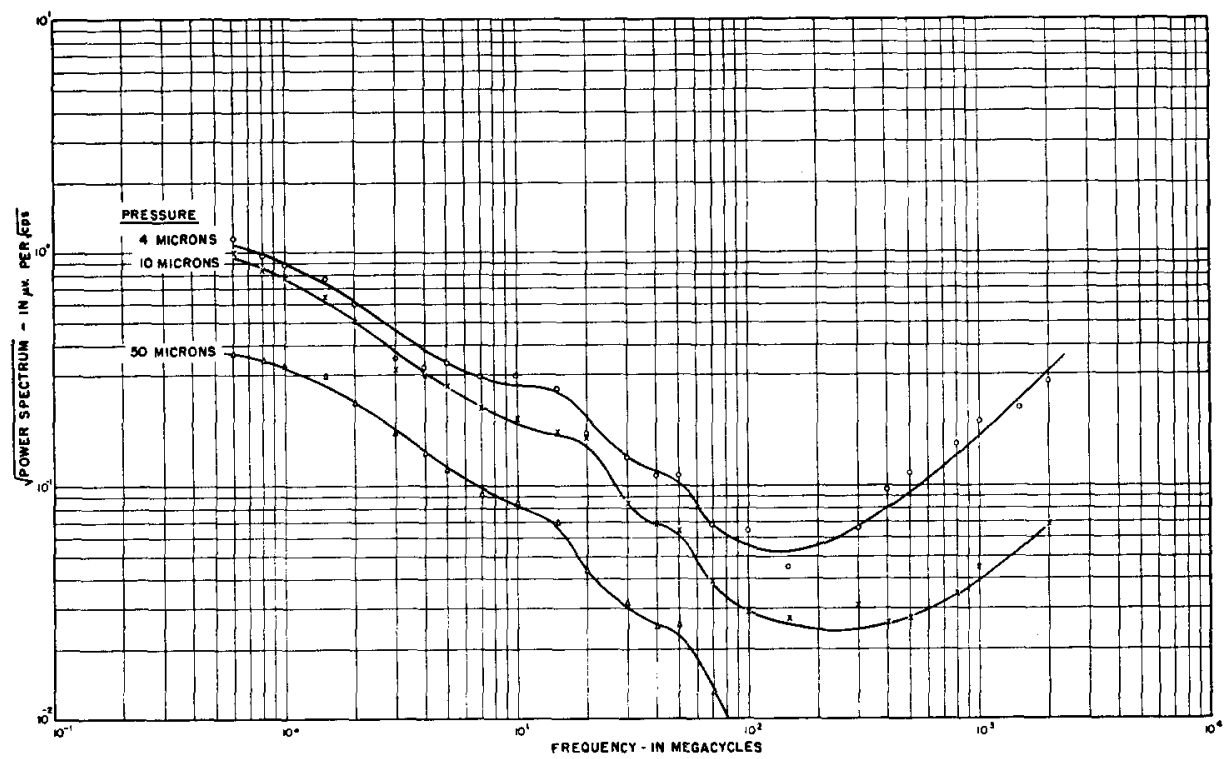

FIG. 5. Spectral distribution of nitrogen discharge in a transverse magnetic field. $B=4550$ gauss

Perhaps this is not surprising, for the higher atomic weight implies an increase in the ion collision frequency relative to the cyclotron frequency, and such an increase would likely dampen any pronounced effect at the cyclotron frequency. Probably this is the reason Cobine in his extensive studies did not report any peculiar behavior of the spectrum at the ion cyclotron frequency (3). Most of his work was with heavier gases than hydrogen and helium; he also used higher pressures and lower magnetic fields than considered here and this would increase the damping at the cyclotron frequency.

Figure 5 shows data taken for nitrogen over a broad frequency range. The ion cyclotron frequency for nitrogen is less than half a megacycle per second and the characteristic dip does not appear in the figure. The absolute calibration on the ordinate is not too significant 
since it depends on the probe size, but some idea of what the numerical value of the ordinate implies is given by noting that the thermal noise from a matched resistance at room temperature would plot as a horizontal line in Fig. 5 with an intercept at $4.5 \times 10^{-4}$. This value is far less than the spectral level represented in the figure. The curves of Fig. 5 show a very broad spectrum, high in intensity compared with thermal noise. Careful investigation showed that there were no

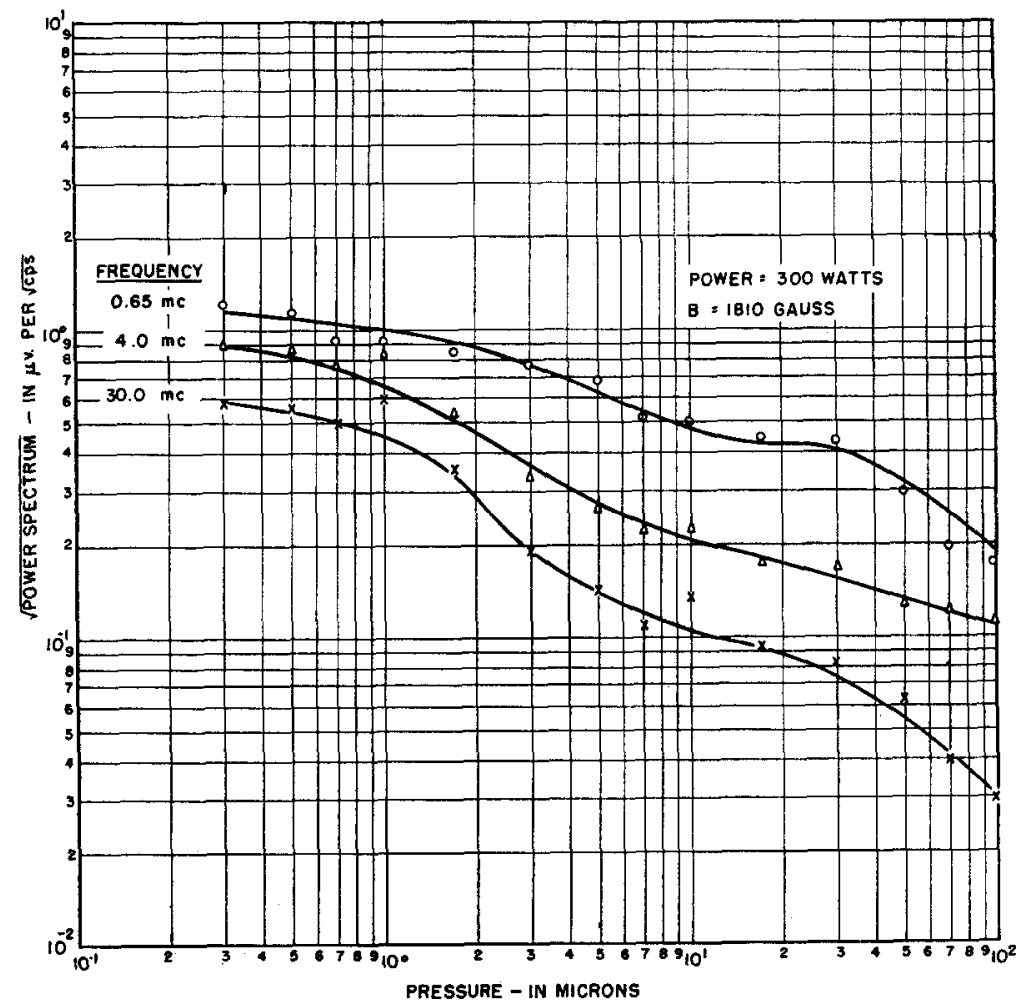

Fig. 6. Spectral distribution for nitrogen as a function of pressure with constant power input.

isolated resonances in this broad frequency range. Varying the magnetic field strength throughout the 1000- to 5000-gauss range did not affect the character of the spectrum. For values of magnetic field less than a few hundred gauss the diffusivity and other visually observable characteristics of the discharge changed considerably. The cathode behavior became erratic, and reliable data would have been difficult to obtain.

The influence of pressure is shown prominently in Fig. 5. An increase in pressure decreases the power spectrum at all frequencies. This result was checked over a wide range of pressures for particular frequencies. The result is shown in Fig. 6. The lowest pressures of 
these curves represent the least values of pressures at which the discharge could be sustained for the fixed power input. JThese curves

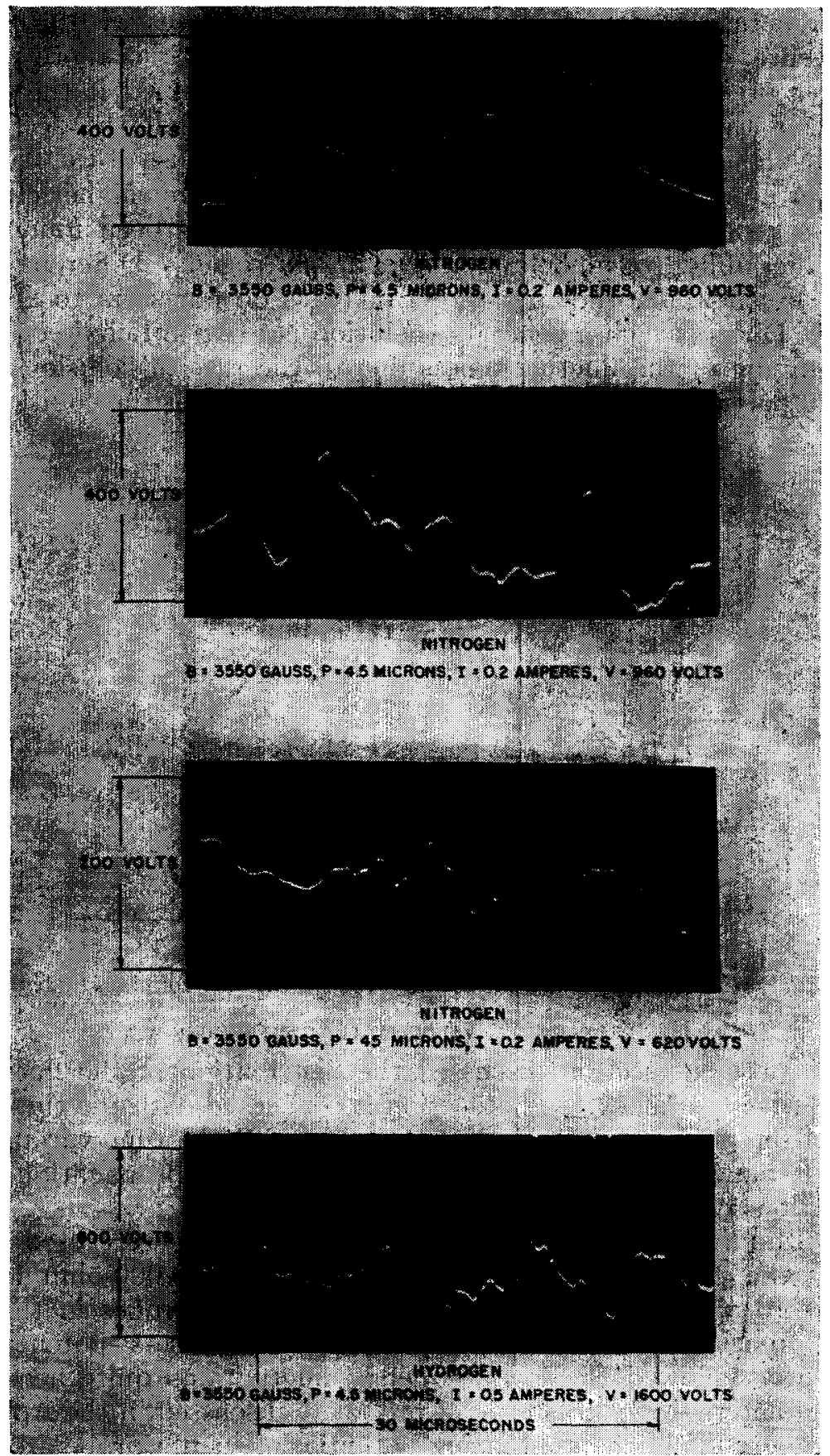

FIg. 7. Potential fluctuations on a probe in a gas discharge. 
indicate that over a wide pressure range a decrease in pressure causes an increase in the spectral energy.

The data were taken at a constant power input to emphasize that the percentage of power converted to noise increases with a decrease in pressure. Thus, the device as a noise generator is more efficient at lower pressures. Similar curves would be obtained if the current instead of the power were kept constant, except that the decrease in noise at higher pressures would be exaggerated because of the reduction of power input at the higher values of pressure. This higher efficiency of noise generation with reduction of pressure has been found in all the studies of hydrogen, helium, argon, and nitrogen. Different probes, magnetic fields, or other parameter changes do not seem to alter this result. Perhaps such a general conclusion is to be anticipated because the damping of the fluctuations is due to the rate of collisions of the charged particles, and a reduction in pressure reduces the collision rate.

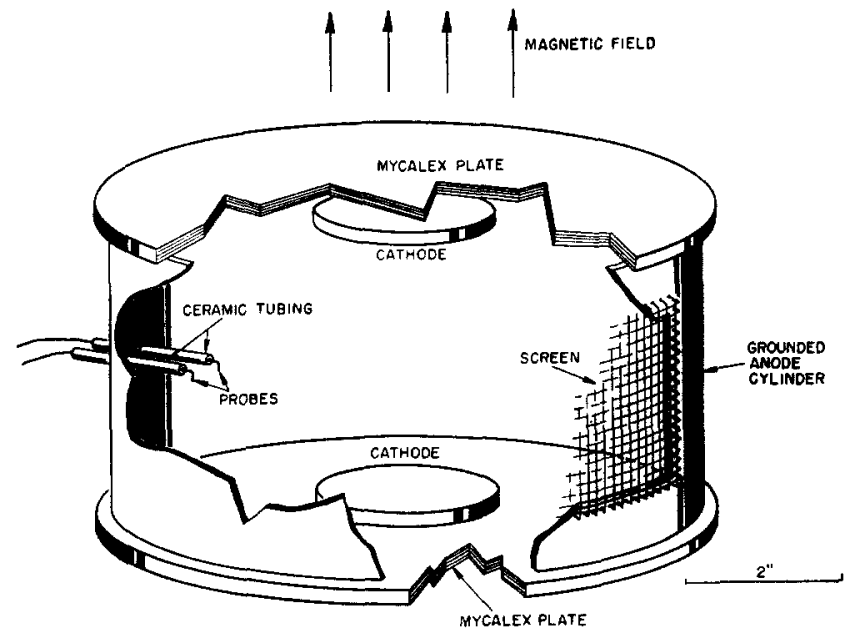

FIG. 8. Electrode structure and probe arrangement for the study of propagation velocity.

PROPAGATION VELOCITY AT LOW FREQUENCY

The previous section discussed the distribution of the fluctuating energy with frequency. This section is concerned with the time variations of the plasma fluctuations. By comparing the time variations on two different probes, the speed and direction of propagation of the fluctuations can be determined.

For the low frequency studies, bare wire probes were used. Electrically the probes were connected through a 300,000-ohm load resistance to a positive potential. By biasing the probes in this way, their potentials remained slightly above the plasma potential and apparently followed the low frequency fluctuations quite accurately. The characteristics of this type of probe are discussed in the Appendix.

Photographs of the fluctuating potential of such a probe are shown 
in Fig. 7. The high intensity and random nature of this disturbance is particularly interesting. If a second probe is placed in the discharge 1 in. from the first and at the same distance from the anode, as indicated in Fig. 8, the fluctuations on the two probes typically have the appearance shown in Fig. 9. The corresponding potentials on the two probes

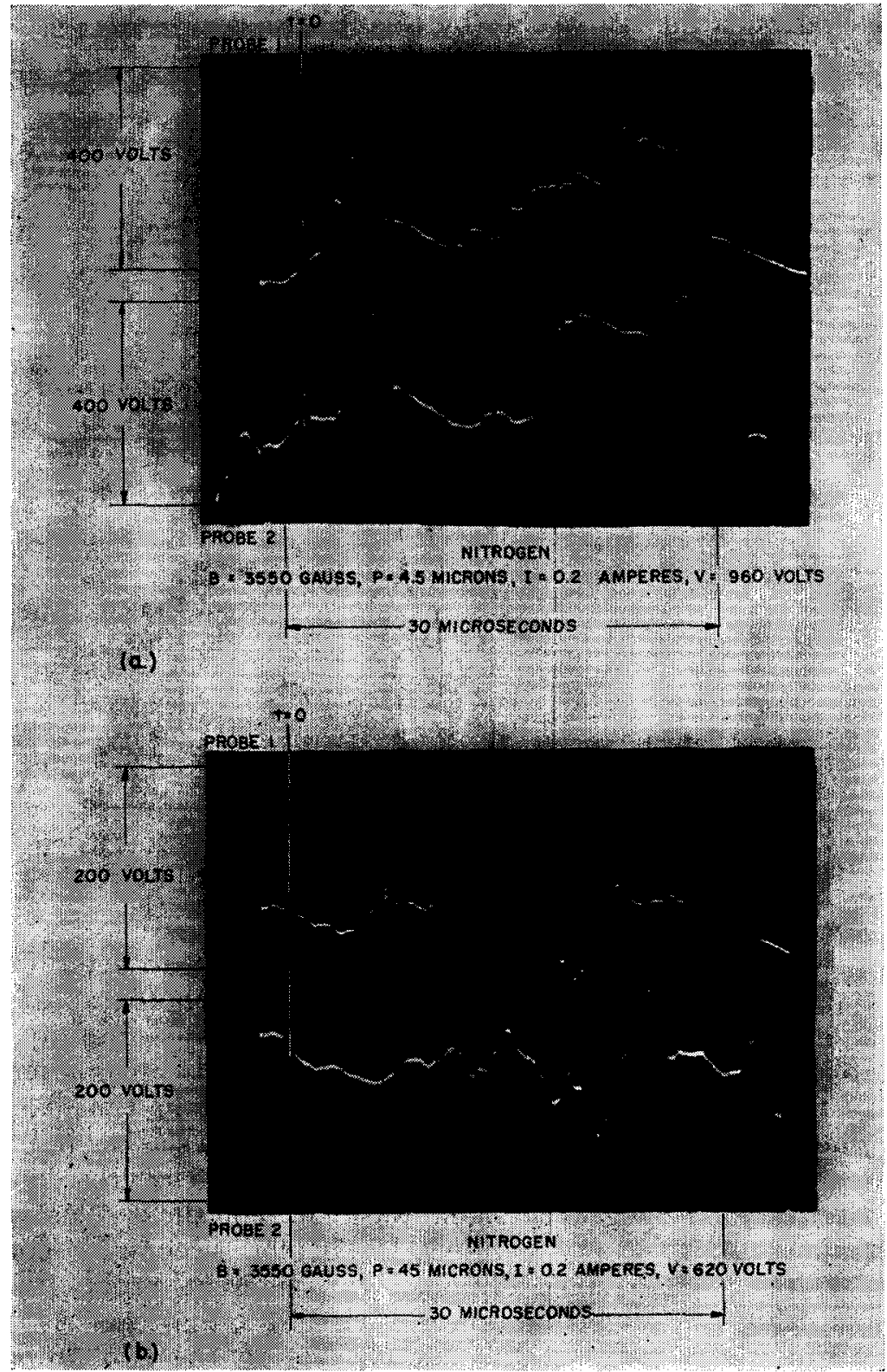

FIG. 9. Potential fluctuations on two neighboring probes. 
appear remarkably alike, with two important exceptions: (1) the fine structure is different, (2) the voltage on probe 2 is delayed in time with respect to the voltage on probe 1 . This delay is related to the time it takes the fluctuation to travel from one probe to the other. If the line from one probe to the other is the direction of propagation, then the velocity can evidently be determined merely by dividing the distance between the two probes by this time delay.

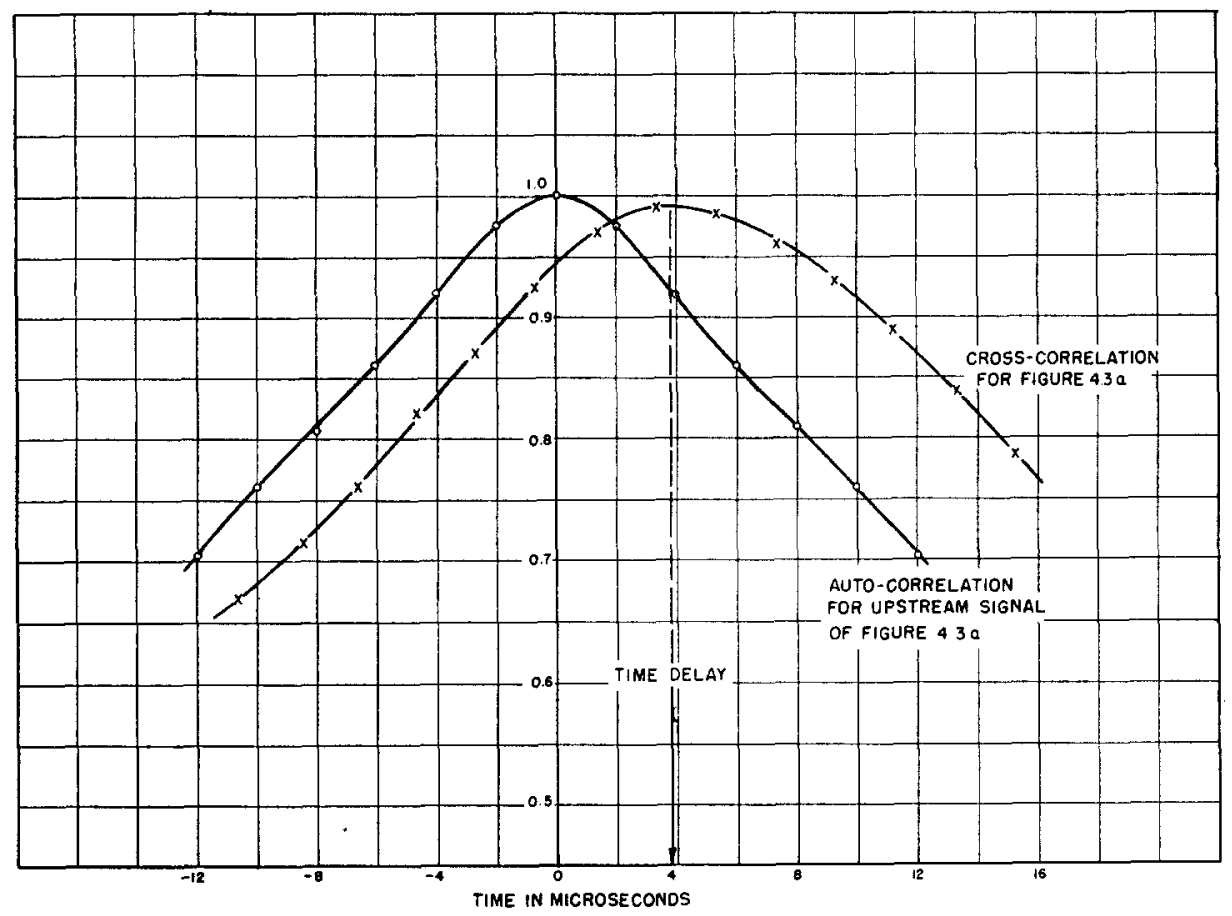

FIG. 10. Representative curves of auto-correlation and cross-correlation used to determine the correlation velocity.

Data were obtained photographically as in Fig. 9 to determine this velocity, except that a reticule appears on the photographs of the working data and the average level of the voltage was carefully noted. These photographs were taken with Polaroid Land cameras mounted on two Tektronix 513D oscilloscopes. The common time base was determined by intensity modulating both beams at the point $t=0$ as indicated in Fig. 9. The sweep-rates were set at 4 microseconds per centimeter. A half-megacycle signal was placed on the oscilloscope and photographed so that any nonlinearity in the sweep-rate could be calibrated out. Data were obtained for different probe positions, magnetic fields, pressures, and power inputs.

The time delay between the fluctuations on the two probes was determined by cross-correlating the two potentials. That is, calling the potential on probe $1, \phi_{1}(t)$, and that on probe $2, \phi_{2}(t)$, the value of 
$\tau$ for which the correlation function $\overline{\phi_{2}(t+\tau) \phi_{1}(t)}$ reached its maximum was taken as the delay. The bar over this product means the average in time, (the average is extended over all time where the potentials are defined by the photographs). Figure 10 shows the cross-correlation

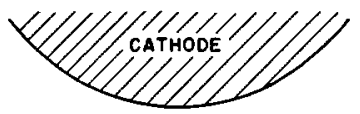

STANDARD CONDITIONS:

$B=3550$ GAUSS

$1 \cdot 0.2$ AMPERES

$p=4.5$ MICRONS

INCREASED CURRENT: I 0.5 AMPERES

INCREASED PRESSURE; $P=45$ MICRONS

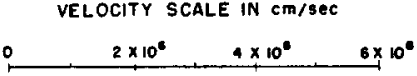

DECREASED FIELD: $B=1870$ GAUSS

THE LOAEMTZ VELOCITY IS THE

AVERAGE ELECTRIC FIELD DIVIOED

BY THE MAGNETIC FIELD

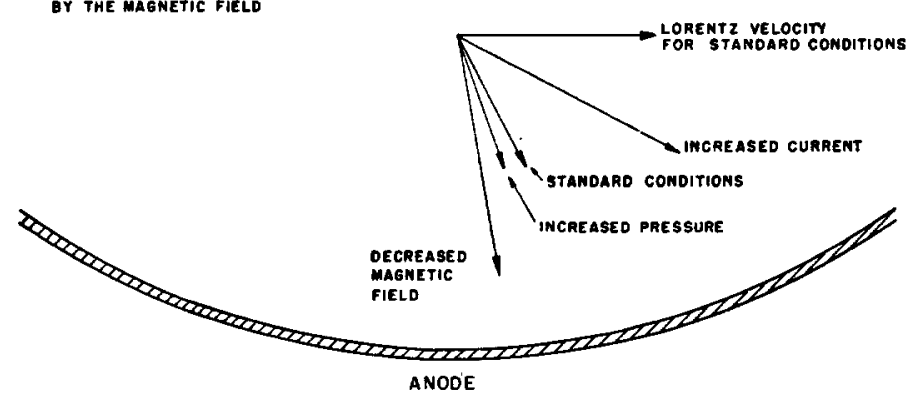

FIG. 11. Vectors showing the direction and amplitude of the propagation velocity.

for the pair of curves given in Fig. $9 a$, and also the auto-correlation for the potential on probe 1 of the same figure. In this case, the correlation functions have been normalized to unity in the usual way, that is,

$$
\begin{aligned}
\text { cross-correlation } & =\frac{\overline{\phi_{2}(t+\tau) \phi_{1}(t)}}{\sqrt{\overline{\phi_{1}{ }^{2}(t)} \overline{\phi_{2}{ }^{2}(t)}}} \\
\text { auto-correlation } & =\frac{\frac{\phi_{1}(t+\tau) \phi_{1}(t)}{\overline{\phi_{1}{ }^{2}(t)}}}{.} .
\end{aligned}
$$

The fact that the cross-correlation of Fig. 10 reaches a value of 0.99 indicated that apart from the delay of 3.8 microseconds the potentials of Fig. $9 a$ are nearly the same.

Figure 11 summarizes the important results of the velocity measurements. The figure shows the direction and magnitude of the velocity of propagation in nitrogen gas with various conditions of operation. 
It is seen that this velocity is the same order of magnitude as the Lorentz drift velocity and is directed in the same quadrant as the electron drift velocity. This suggests that the fluctuations propagate with approximately the average electron velocity. If this is true it supports one hypothesis which requires that the fluctuations amplify as they propagate, in a manner analogous to that found in a trarelingwave tube.

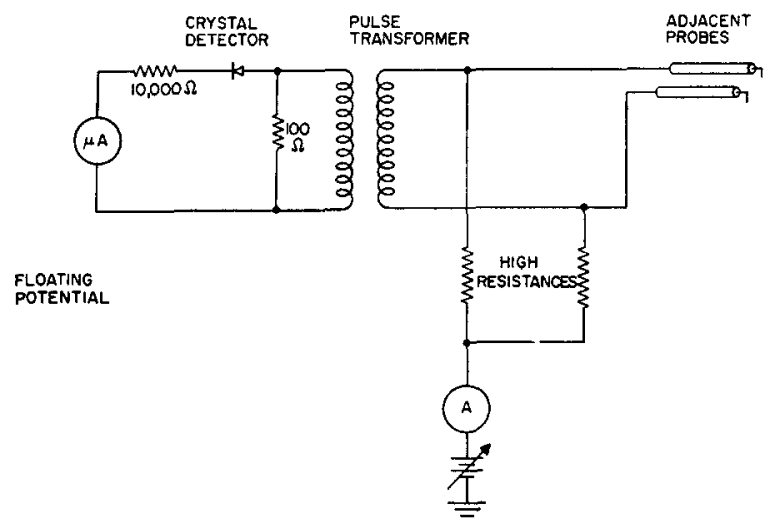

FIG. 12. Circuit for measuring amplification with propagation.

THE AMPLIFICATION WITH PROPAGATION

In order to test the assumption that the fluctuations can be identified with waves that amplify as they propagate, a special type of double probe experiment was devised. With the probes of the previous section, the fluctuating potentials were measured with respect to the grounded anode and hence included the effects of voltage gradients in the vicinity of the anode as well as gradients in the immediate vicinity of the probe. To measure only the local intensity of a disturbance, it was desirable to design a probe system that would respond only to potential gradients in a localized region. This was accomplished by using two adjacent probes connected to a small pulse transformer which served to isolate the probes from ground and also acted as a differential input transformer. Figure 12 is a schematic diagram of the circuit. The two probes were connected to opposite ends of the transformer primary, and only the voltage between the two probes produced a voltage in the secondary. Biasing currents for the two probes were supplied through a resistance which was high enough to minimize any shunt loading. This biasing current was maintained constant as the probes were moved to different points in the discharge.

Capacitance between the probes and ground (through the transformer) was reduced by "floating" the transformer core, crystal detector, resistance, and the microammeter with respect to ground. The circuit was tested with a signal generator, and it was shown that the output depended only on the differential input at the probes. 
The data of this section are shown in Fig. 13, where the voltage difference between the probes is plotted as a function of the distance from the probes to the cathode. The operating pressure, magnetic field and current are the same as the standard conditions given in Fig. 11. The curve of Fig. 13 is hardly conclusive evidence for gain, but in the region between cathode and anode there is an apparent gain of about $5 \mathrm{db}$. per inch. This supports the hypothesis that the noise fluctuations amplify as they propagate.

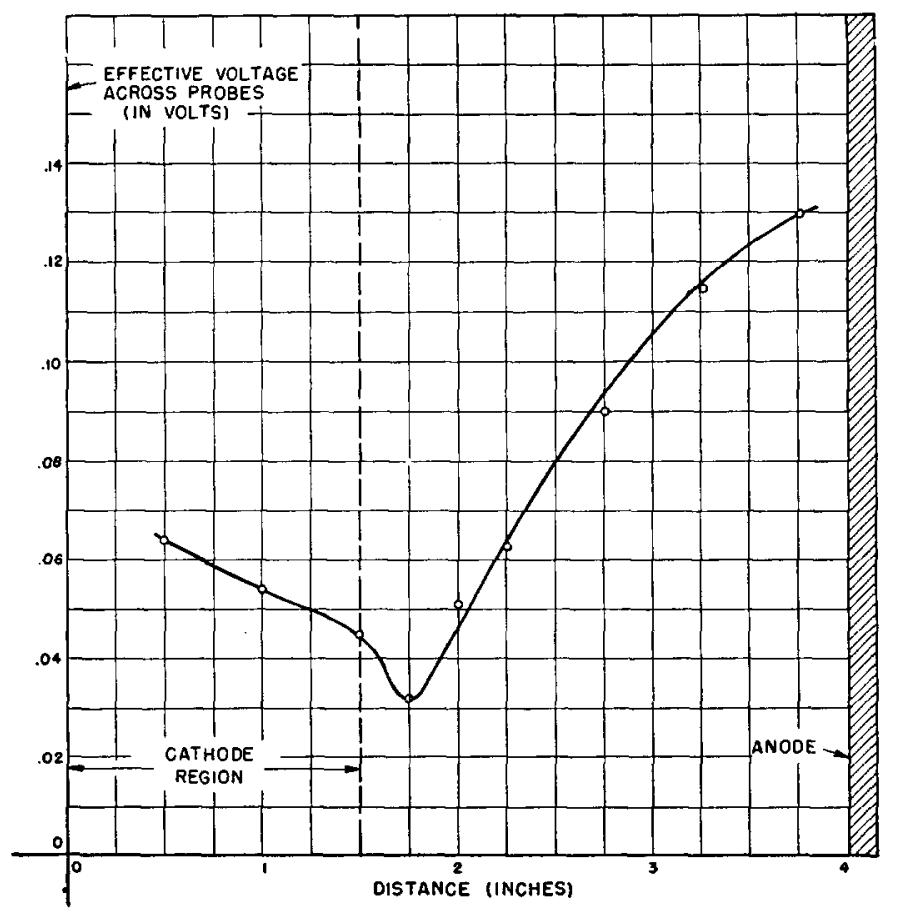

FIG. 13. Curve showing the amplitude of fluctuations between probes as a function of the position of the probes.

CONCLUSION

High-amplitude, plasma fluctuations characterize an electrical discharge in a transverse magnetic field. These fluctuations are hundreds of volts in magnitude and extend from d.c. to the microwave frequencies. The spectrum is principally due to electron disturbances, but ions make a significant contribution near the ion cyclotron frequency, especially for the light gases. The fluctuations appear to propagate in the direction of the electron drift and approximately at the electron drift velocity. There is evidence to suggest that these fluctuations amplify as they propagate.

Acknowledgment-The authors wish to acknowledge the assistance of Professors W. G. Dow and G. Hok during many phases of this study. 


\section{REFERENCES}

(1) H. W. Batten and H. C. Early, "Plasma Fluctuations in Crossed Electric and Magnetic Fields," Technical Report No. 2, Engineering Research Institute, University of Michigan, Office of Ordnance Research Contract No. DA-20-018-ORD-11913, May 1954.

(2) D. Gabor, "Plasma Oscillations," Brit. Jour. of A pp. Phy., Vol. 22, p. 209 (1951).

(3) J. D. Cobine and C. J. Gallagher, "Noise and Oscillations in Hot Cathode Arcs," Jour. Franklin InST., Vol. 243, p. 41 (1947); and "Effects of a Magnetic Field on Oscillations and Noise in Hot-Cathode Arcs," J. Appl. Phys., Vol. 18, p. 110 (1947).

(4) H. C. Early and W. G. Dow, "Supersonic Wind at Low Pressures Produced by Arc in Magnetic Field," Phys. Rev., Vol. 79, p. 186 (1950).

(5) H. C. Early, H. L. Smith and D. C. Lu, "Electrical Wind Phenomena," Summary Report No. 1, Engineering Research Institute, University of Michigan, Office of Ordnance Research Contract No. DA-20-018-ORD-11913, November 1952.

(6) S. Ballantine, "Fluctuation Noise Due to Collision Ionization in Electronic Amplifier Tubes," Physics, Vol. 4, p. 294 (1933); and Cobine and Gallagher, op. cit.

(7) W. D. McBee and W. G. Dow, "The Influence of a Transverse Magnetic Field on an Unconfined Glow Discharge," Trans. A. I. E. E., Vol. 72, Pt. I, pp. 229-238 (1953).

\section{Probe Design}

\section{APPENDIX}

An ideal plasma probe should sample the fluctuating electrical potential at a given point in the plasma without disturbing the phenomena being sampled. To achieve this, the probe should be as small as possible, and the load impedance due to the associated circuitry should be high enough so that the probe is free to "ride along" with the potential fluctuations. However, in any realizable probe design, the effects of stray capacitance and load impedance become quite serious. The two designs which were used in this investigation fall short of what might have been desired and represent compromises of the ideal probe requirements.

The probe shown in Fig. 1 used a hypodermic needle covered with a glass sleeve. The wire passing through the needle formed a coaxial line having the same impedance as the coaxial cable to which it was connected. This arrangement had the advantage that the load impedance of the terminated coaxial line was constant at all frequencies. Since the cable impedance of $\mathbf{5 0}$ ohms was objectionably low, the probe was covered with an insulating glass sleeve which reduced the tendency of the probe to disturb the "waves" propagating through the plasma. Of course, this capacitance type of coupling through the glass was frequency sensitive, and caused a decrease in the response at the low frequency end of the power spectrum.

The "bare" wire probe shown in Fig. 8 was used for the propagation studies at low frequencies where the signal from the glass sleeve probe was too low. Instead of using a terminated coaxial line, the probe was connected directly to the high impedance input of an oscilloscope and all stray capacitance was reduced to a minimum. The total capacitance to ground including the oscilloscope input was not over $25 \mu \mu f d$.

An "exposed" wire probe of this type in a gas discharge plasma tends to act as a diode rectifier since the electron current associated with a positive potential is many times larger than the ion current for a negative potential. Hence, unless suitable precautions are taken, the probe behaves as a diode peak reading voltmeter, accepting electrons only on the negative peaks of plasma voltage swing and maintaining an average $d$-c. potential which is perhaps 50 to 100 volts more negative than the average potential of the surrounding plasma (7). Since the voltage on such a probe would be a poor reproduction of the plasma fluctuations, it was found desirable to connect the probe through a high load resistance to a positive bias voltage of several hundred volts so that a continuous electron current was drawn from the plasma. When biased in this manner, the probe tends to maintain a potential slightly positive with respect to the instantaneous plasma potential and apparently follows the low frequency fluctuations rather accurately. 